\title{
Flow Behaviour of Labeled Red Blood Cells in Microchannels : a confocal micro-PTV assessment
}

\author{
R. Lima ${ }^{1,2}$ \\ ${ }^{1}$ DTM, ESTiG/IPB, Institute Polytechnic of Braganca, C. Sta. Apolonia, 5301-857 Braganca, Portugal; \\ ${ }^{2}$ CEFT, FEUP, University of Porto, R. Dr. Roberto Frias, 4200-465 Porto, Portugal..
}

\begin{abstract}
The development of optical experimental techniques has contributed to obtain explanations on the blood flow behaviour through microvessels. Although the past results have been valuable, detailed studies on the flow properties of blood in the microcirculation have been limited by several technical factors such as poor spatial resolution and difficulty to obtain quantitative detailed measurements at such small scales. In recent years, due to advances in computers, optics, and digital image processing techniques, it has become possible to combine particle tracking methods with confocal microscopes. As a result, this combination has greatly increased the resolution of the conventional PIV systems and consequently provided additional detailed description on the blood cells motion not obtainable by traditional methods. In this work, we will show an overview of the most recent results on the blood flow behaviour in microchannels obtained by state-of-the-art confocal micro-PIV/PTV system.
\end{abstract}

Keywords- Microcirculation, red blood cells, microchannels, confocal micro-PTV, radial dispersion

\section{INTRODUCTION}

Blood flow in both microvessels and microchannels has been measured by several measurements techniques such as: double-slit photometric [1], laser-Doppler anemometer $[2,3]$, video-based methods $[4,5]$. Although the past research fondinds have been encouraging, detailed studies on the way blood flow behaves at a microscopic level have been limited by several factors such as poor spatial resolution, difficulty to obtain accurate measurements at such small scales, optical errors arisen from walls of the microvessels, high concentration of blood cells, and difficulty in visualization of results due to insufficient computing power and absence of reliable image analysis techniques. In recent years, due to advances in computers, optics, highspeed imaging and image processing techniques, it has become possible to combine a conventional particle image velocimetry (PIV) system with an inverted microscope and consequently improve both spatial and temporal resolution [6-10]. This system, known as micro-PIV (see Fig.1), has been applied to study the flow properties of blood in both microvessels and microchannels $[6,7,9,10]$. Although, micro-PIV systems are gaining widespread among the biomicrofluidics community due to its high spatial and temporal resolution, the employment of conventional microscope leads to the entire illumination of the flow field resulting in high levels of background noise and consequently errors on the flow measurements [11]. These errors can be partially removed by using a spinning disk confocal microscope (SDCM) [12, 13]. Due to its spatial filtering technique and multiple point light illumination system, confocal micro-PIV [14 - 16] has become accepted as a reliable method for measuring in vitro blood flow through microchannels. The current mini review will show a short overview of the most relevant results on the blood flow behaviour in microchannels obtained by our confocal micro-particle tracking velocimetry (PTV) system.

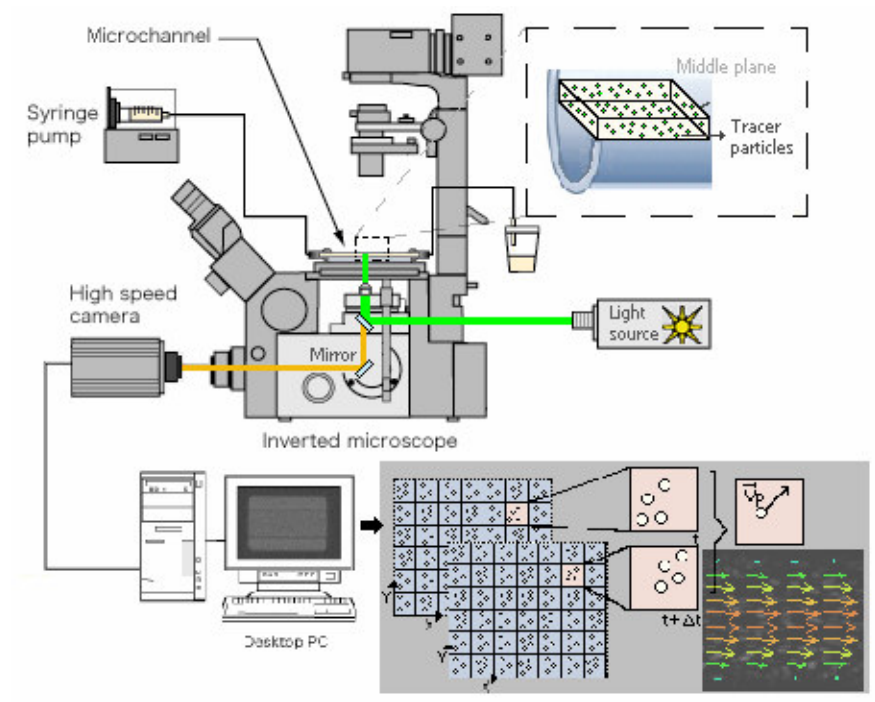

Fig. 1 Conventional micro-PIV system (adapted from [19]).

\section{CONFOCAL MICRO-PTV SYSTEM}

\section{Working fluids and RBC labeling}

Experiments were carried out with different working fluids: dextran 40 (Dx-40; Otsuka Medicine, Tokyo, Japan) containing about $3 \%$ hematocrit (Hct) up to $35 \% \mathrm{Hct}$ of human red blood cells (RBCs). The RBCs were labeled with a lipophilic carbocyanime derivative, chloromethylben- 
zamido (CM-Dil, C-7000, Molecular Probes, Eugene, OR, USA). This cell tracker was selected due to its strong photostable fluorescence, excellent cellular retention, and minimal cytotoxicity. A full description about the procedure for labeling the human RBCs can be found at Lima et al. 2009 [17].

\section{Microchannels}

A $100-\mu \mathrm{m}$ and $50-\mu \mathrm{m}$ circular borosilicate glass microchannel was fabricated by Vitrocom (Mountain Lakes, NJ, USA). The microchannel was mounted on a slide glass approximately $80 \mu \mathrm{m}$ thick and immersed in glycerol that had the same refractive index to minimize refraction from the walls. Additionally, by using a wire casting technique [18] it was possible to fabricate $75-\mu \mathrm{m}$ circular polydimensiloxane (PDMS) microchannels.

\section{Experimental setup}

The confocal micro-PTV system consisted of an inverted microscope (IX71; Olympus, Tokyo, Japan) combined with a confocal scanning unit (CSU22; Yokogawa, Tokyo, Japan), a diode-pumped solid state (DPSS) laser (Laser Quantum Ltd., Stockport, UK) and a high-speed camera (Phantom v7.1; Vision Research, NJ, USA). The microchannels were placed on the stage of the inverted microscope where the flow rate of the working fluids was kept constant using a syringe pump (KD Scientific Inc., Holliston, MA, USA). A thermo-plate controller (Tokai Hit, Shizuoka, Japan) was employed to apply a temperature around the microchannel of about $37^{\circ} \mathrm{C}$. More detailed information about this system can be found elsewhere [14, 17, 19].

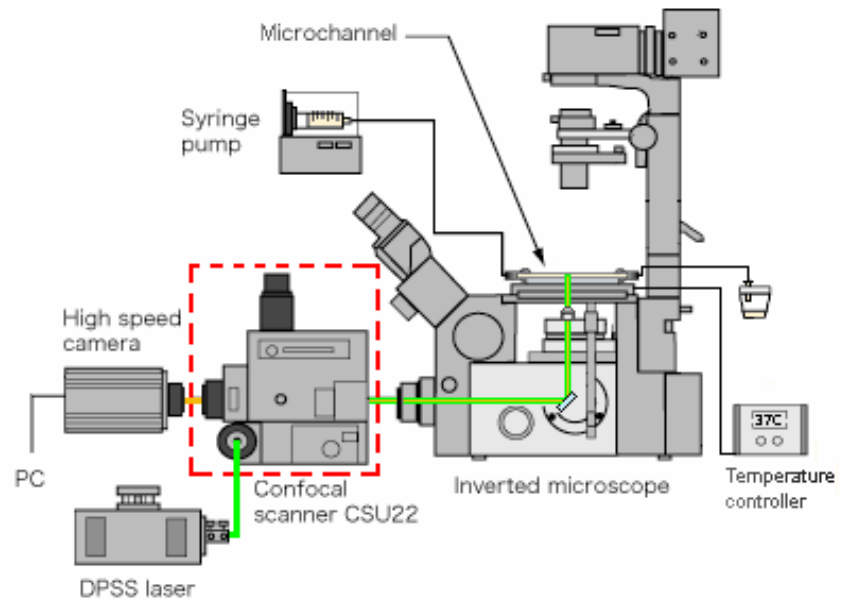

Fig. 2 Confocal micro-PTV experimental set-up (adapted from 17).

\section{Tracking RBC trajectory}

The recorded series of $x y$ confocal images were evaluated in Image $\mathbf{J}$ [20] (NIH, Bethesda, MD, USA) using the manual tracking MtrackJ [21] plug-in. Generally, most of RBCs were followed for periods of time from $0.34 \mathrm{~s}$ up to $1 \mathrm{~s}$, with temporal resolution of $10 \mathrm{~ms}$. The motion of the labeled RBCs, they were manually tracked through successive images using the bright centroid criteria available at the MtrackJ. Using this method, it was possible to track labeled RBCs even when two cells were in near proximity. After obtaining series of $x$ and $y$ positions, data were exported for the determination of physical quantities such as velocity, radial displacement, and dispersion coefficient.

\section{CONFOCAl MiCRO-PTV RESUlts \& DISCUSSION}

First confocal microscopy experiments with in vitro blood were carried out in both glass [14, 15] and PDMS [16] microchannels. In these research woks measurements of both ensemble and instantaneous velocity profiles for in vitro blood with Hcts up to $20 \%$ were performed. Although the ensemble velocity profiles were in close agreement with a theoretical model, some fluctuations in the instantaneous velocity profiles were found to be closely related to the increase in the Hct implying that the presence of RBCs within the plasma flow influences the measurements of the instantaneous velocity fields. Moreover, for the case of glass microchannels and for Hct bigger than 9\%, the light absorbed by the RBCs contributes to diminish the concentration of tracer particles in the recorded confocal images. This low density images become more evident for Hct bigger than $20 \%$, which generates spurious errors in the velocity fields. As a result, Lima and his colleagues [17, 19, 22] have applied a new approach, known as confocal microPTV, to track the trajectories of individual labeled RBCs at high Hcts. Figures 3 and 4 show the ability of this confocal method to measure the motion of blood cells at both diluted and high suspensions of RBCs, respectively. Figure 5 shows trajectories of labeled RBCs in a PDMS microcannel.

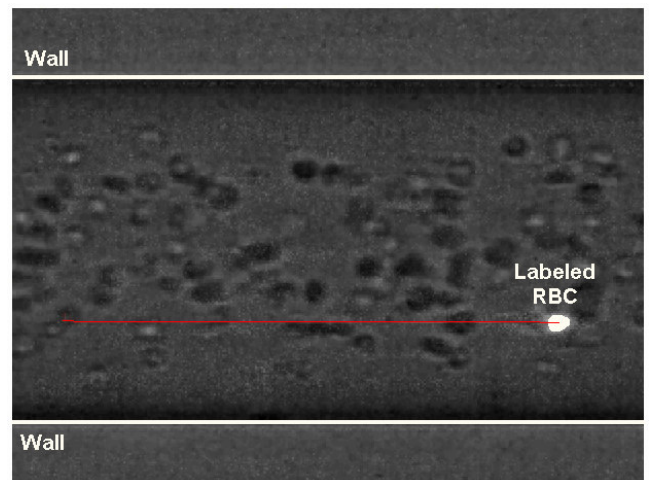

Fig. 3 Trajectories of labeled RBCs at diluted suspensions of cells obtained by the proposed confocal micro-PTV system (adapted from [22] ). 


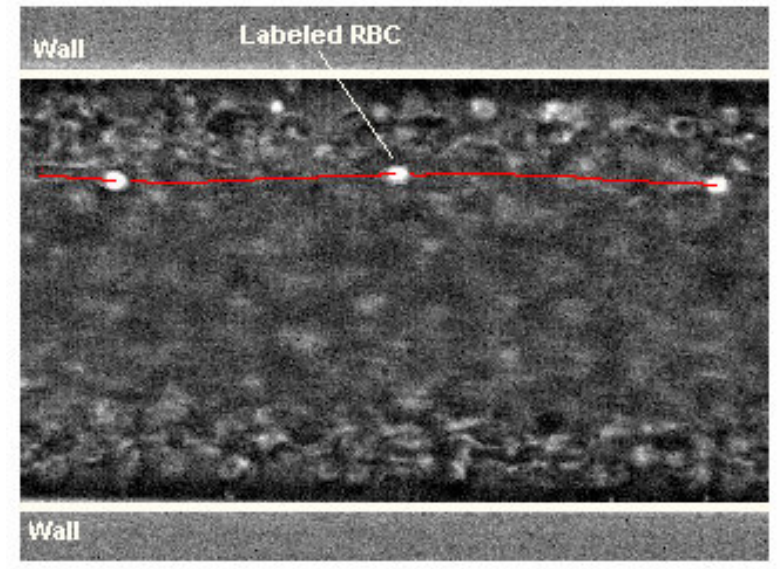

Fig. 4 Trajectories of labeled RBCs at high suspensions of cells obtained by the proposed confocal micro-PTV system. Experiments were carried out in glass microchannels (adapted from [22]).

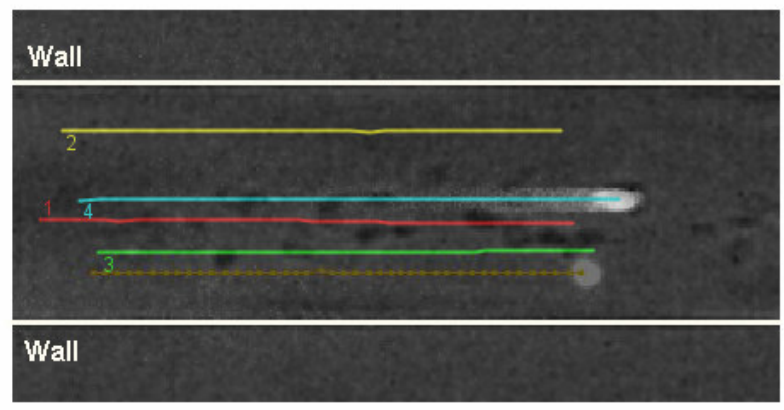

a)

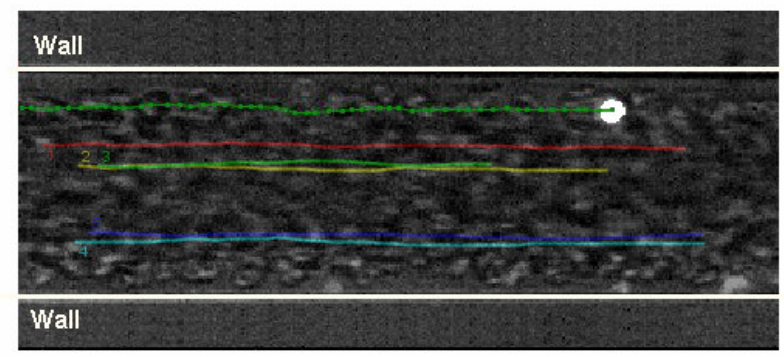

b)

Fig. 5 Trajectories of labeled RBCs at a) diluted and b) high suspensions of cells obtained by the proposed confocal micro-PTV system. Experiments were carried out in PDMS circular microchannels (adapted from [18]).

The capacity of the confocal system to generate thin infocus planes has allowed both qualitative and quantitative measurements in flowing blood at concentrated suspensions of: cell-cell hydrodynamic interaction, $\mathrm{RBC}$ orientation and $\mathrm{RBC}$ radial dispersion at different depths [17, 22]. Furthermore RBCs radial dispersion coefficient $\left(D_{y y}\right)$ were determined in the middle plane of $100 \mu \mathrm{m}$ capillaries [22]. The results demonstrated that $\mathrm{RBCs} D_{y y}$ tends to increase with the Hct. For instance Fig.6 shows clear evidence that RBCs
$D_{y y}$ at Hct of $24 \%$ is almost one order magnitude bigger than $D_{y y}$ with $3 \%$ Hct. Similar qualitative results were also obtained in a $75 \mu \mathrm{m}$ PDMS circular microchannel (see Fig.7). These research findings show evidence that the RBCs flowing in a crowded environment tend to undergo multi-body collisions which increases the amplitude of the RBC's lateral motion and consequently RBC $D_{y y}$. Hence, $\mathrm{RBCs}$ at high concentrations tend to exhibit higher erratic radial displacement compared to dilute suspensions of RBCs.

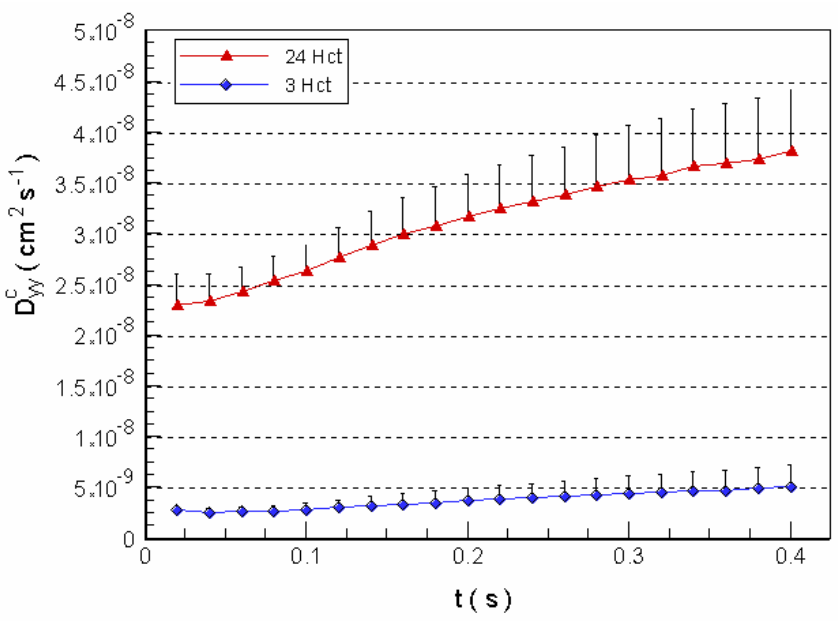

Fig. 6 RBCs radial dispersion coefficient $\left(D_{y y}\right)$ of $100 \mu \mathrm{m}$ glass microchannel for $3 \%$ Hct and $24 \%$ Hct. (adapted from [22] ).

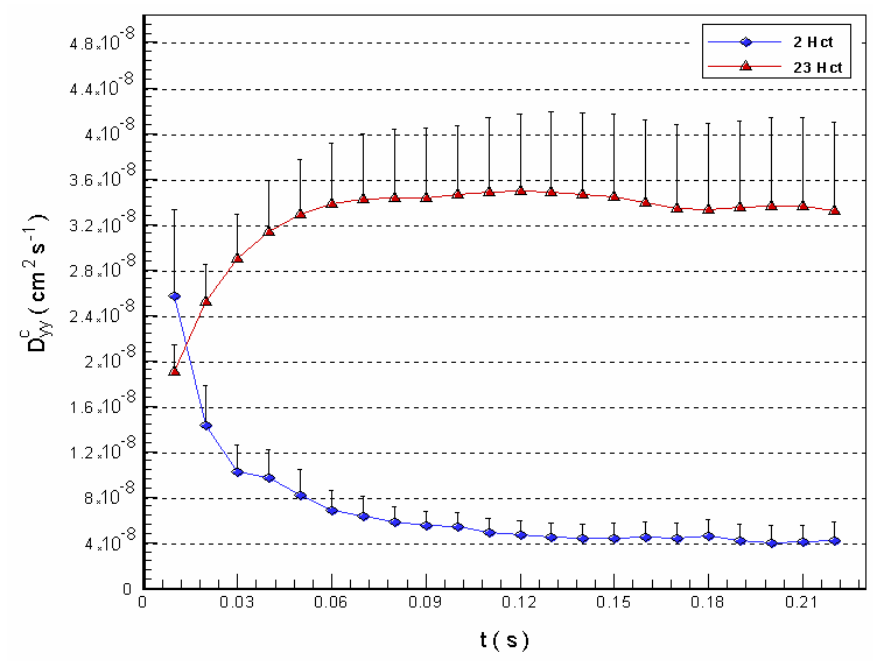

Fig. 7 RBCs radial dispersion coefficient $\left(D_{y y}\right)$ of $75 \mu \mathrm{m}$ PDMS circular microchannel for $2 \% \mathrm{Hct}$ and $23 \%$ Hct. (adapted from [18] ).

\section{Conclusion \& FUture DiReCTIOS}

Owing to its optical sectioning ability and consequent improvement of the image contrast and definition, the proposed confocal micro-PTV system has proved to be a powerful technique to provide detailed quantitative measure- 
ments on the motion of RBCs cells at both diluted and high suspensions of cells. The results suggest that the RBC paths are strongly dependent on the Hct and as a result the RBC dispersion coefficient tend to increase with the Hct.

The results obtained from the presented study were performed in straight microchannels. In the near future, we expect to measure the RBC's motions in more complex geometries such as bifurcations, contractions and expansions. Moreover, by culturing endothelial cells within the PDMS microfluidic device, we expect to develop a flow system device that closely mimics the in vivo microvessel environment.

\section{ACKNOWLEDGMENT}

This study was supported in part by the following grants: International Doctoral Program in Engineering, from the Ministry of Education, Culture, Sports, Science and Technology of Japan (MEXT), 2007 Global COE Program "Global Nano-Biomedical Engineering Education and Research Network", Japan. Grant-in-Aid for Science and Technology (PTDC/SAU-BEB/108728/2008, PTDC/SAUBEB/105650/2008 and PTDC/EME-MFE/099109/2008) from the Science and Technology Foundation (FCT) and COMPETE, Portugal. The author would like to thank Professor Takami Yamaguchi, Professor Shigeo Wada and Dr. Takuji Ishikawa for their valuable suggestions and discussion during this research work.

\section{REFERENCES}

1. Nash G., Meiselman H., (1983) Red cell and ghost viscoelasticity. Effects of hemoglobin concentration and in vivo aging. Biophysical Journal 43: 63-73.

2. Uijttewaal W., Nijhof E., Heethaar R (1994) Lateral migration of blood cells and microspheres in two-dimensional Poiseuille flow: a laser Doppler study. Journal of Biomechanics 27: 35-42.

3. Golster H., Linden M., Bertuglia S. et al. (1999) Red blood cell velocity and volumetric flow assessment by enhanced high-resolution laser Doppler imaging in separate vessels of hamster cheek pouch microcirculation. Microvascular research 58: 62-73

4. Goldsmith H. (1971) Red cell motions and wall interactions in tube flow, Federation Proceedings 30: 1578-1588.

5. Parthasarathi A., Japee S., Pittman R. (1999) Determination of red blood cell velocity by video shuttering and image analysis. Annals of Biomedical Engineering 27: 313-325

6. Sugii Y, Okuda R, Okamoto K, Madarame H (2005) Velocity measurement of both red blood cells and plasma of in vitro blood flow using high-speed micro PIV technique. Measurement Science and Technology 16: 1126-1130.
7. Nakano A., Sugii Y., Minamiyama M., Niimi H. (2003) Measurement of red cell velocity in microvessels using particle image velocimetry (PIV). Clinical Hemorheology and Microcirculation 29: 445-455.

8. Sugii Y, Nishio S, Okamoto K (2002) In vivo PIV measurement of red blood cell velocity field in microvessels considering mesentery motion. Physiol. Meas. 23:403-416.

9. Vennemann P., K. Kiger, R. Lindken, et al. (2006) In vivo micro particle image velocimetry measurements of blood-plasma in the embryonic avian heart. Journal of Biomechanics 39: 1191-1200.

10. Kim G B, Lee S J (2006) X-ray PIV measurements of blood flows without tracer particles. Experiments in Fluids 41:195-200.

11. Meinhart C, Wereley S., Gray H (2000) Volume illumination for twodimensional particle image velocimetry Measurement Science and Technology 11: 809-814.

12. Tanaani T, Otsuki S, Tomosada N e tal. (2002) High-speed 1frame/ms scanning confocal microscope with a microlens and Nipkow disks. Applied Optics 41: 4704-4708.

13. Inoue, S., Inoue, T. (2002) Direct-view high-speed confocal scanner: the CSU-10. Cell Biological Applications of Confocal Microscopy, Academic Press, 87-127.

14. Lima, R. et al. (2006) Confocal micro-PIV measurements of three dimensional profiles of cell suspension flow in a square microchannel. Measurement Science and Technology 17: 797-808.

15. Lima, R., et al., (2007) In vitro confocal micro-PIV measurements of blood flow in a square microchannel: the effect of the haematocrit on instantaneous velocity profiles. Journal of Biomechanics 40: 27522757.

16. Lima R. et al. (2008) In vitro blood flow in a rectangular PDMS microchannel: experimental observations using a confocal micro-PIV system. Biomedical Microdevices 10: 153-67.

17. Lima, R., et al. (2009) Measurement of individual red blood cell motions under high hematocrit conditions using a confocal microPTV system. Annals of Biomedical Engineering 37: 1546-1559.

18. Lima, R., et al. (2009) Axisymmetric PDMS microchannels for in vitro haemodynamics studies. Biofabrication 1: 035005.

19. Lima, R. Analysis of the blood flow behavior through microchannels by a confocal micro-PIV/PTV system. PhD (Eng.), Bioengineering and Robotics Department, Tohoku University, Sendai, Japan, 2007.

20. Abramoff M, Magelhaes P and Ram S, (2004) Image processing with image. J. Biophotonics Int. 11:36-42.

21. Meijering E, Smal I, Danuser G, (2006) Tracking in molecular bioimaging. IEEE Signal Process. Mag. 23: 46-53.

22. Lima, R., et al. (2008) Radial dispersion of red blood cells in blood flowing through glass capillaries: role of hematocrit and geometry. Journal of Biomechanics 41: 2188-2196.

Author: Rui Lima
Institute: Institute Polytechnic of Braganca
Street: Campus Sta. Apolonia
City: Braganca
Country: Portugal
Email: ruimec@ipb.pt

Author: Rui Lima

City: Braganca

Email: ruimec@ipb.pt 